\title{
Dye-Sensitized Solar Cells
}

\section{for efficient solar and artificial light conversion}

\author{
Carolina Hora,${ }^{\dagger}$ Fátima Santos,${ }^{\dagger}$ M. Goreti F. Sales, ${ }^{\ddagger}$ Dzmitry Ivanou, ${ }^{* \dagger}$, Adélio Mendes ${ }^{*}, \dagger$ \\ ${ }^{\dagger} L E P A B E$, Departamento de Engenharia Química, Faculdade de Engenharia, Universidade do \\ Porto, Rua Dr. Roberto Frias, 4200-465 Porto, Portugal \\ BioMark-CEB/Centro de Engenharia Biológica, Instituto de Engenharia Superior do Porto, Rua \\ António Bernardino de Almeida, 431, 4249-015 Porto, Portugal
}

Number of pages: 5

Number of figures: 7

Number of tables: 0

${ }^{*}$ Corresponding author. Tel.: +351920427795 ;

E-mail address: ivanou@fe.up.pt (Dzmitry Ivanou).

${ }^{*}$ Corresponding author. Tel.: +351 225081695;

E-mail address: mendes@fe.up.pt (Adélio Mendes). 


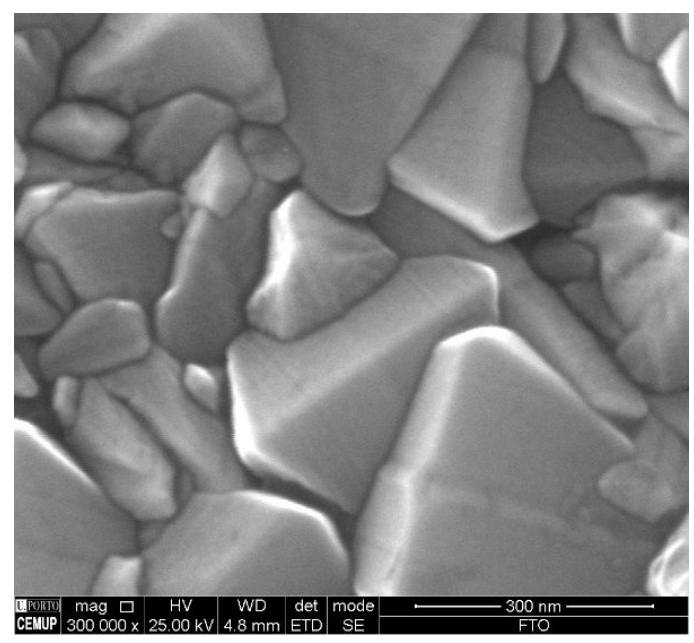

(a)

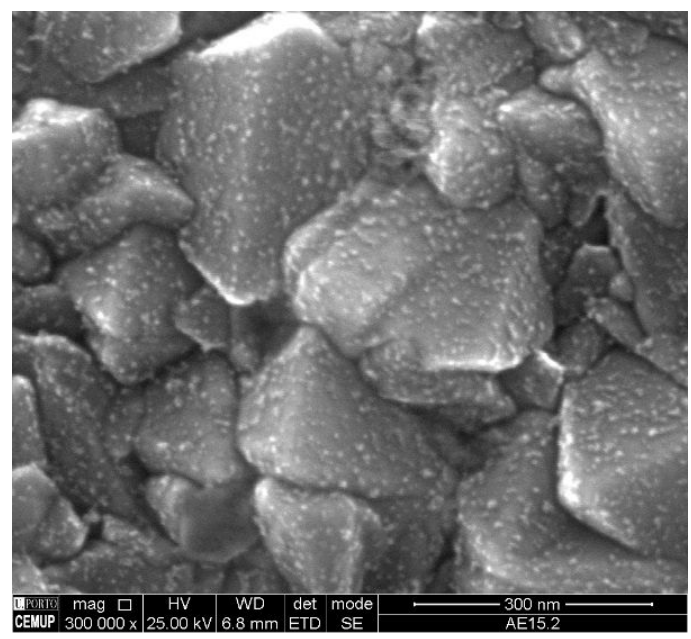

(b)

Figure S1. SEM images of the FTO-glass surface before (a) and after activation with Pt (b).

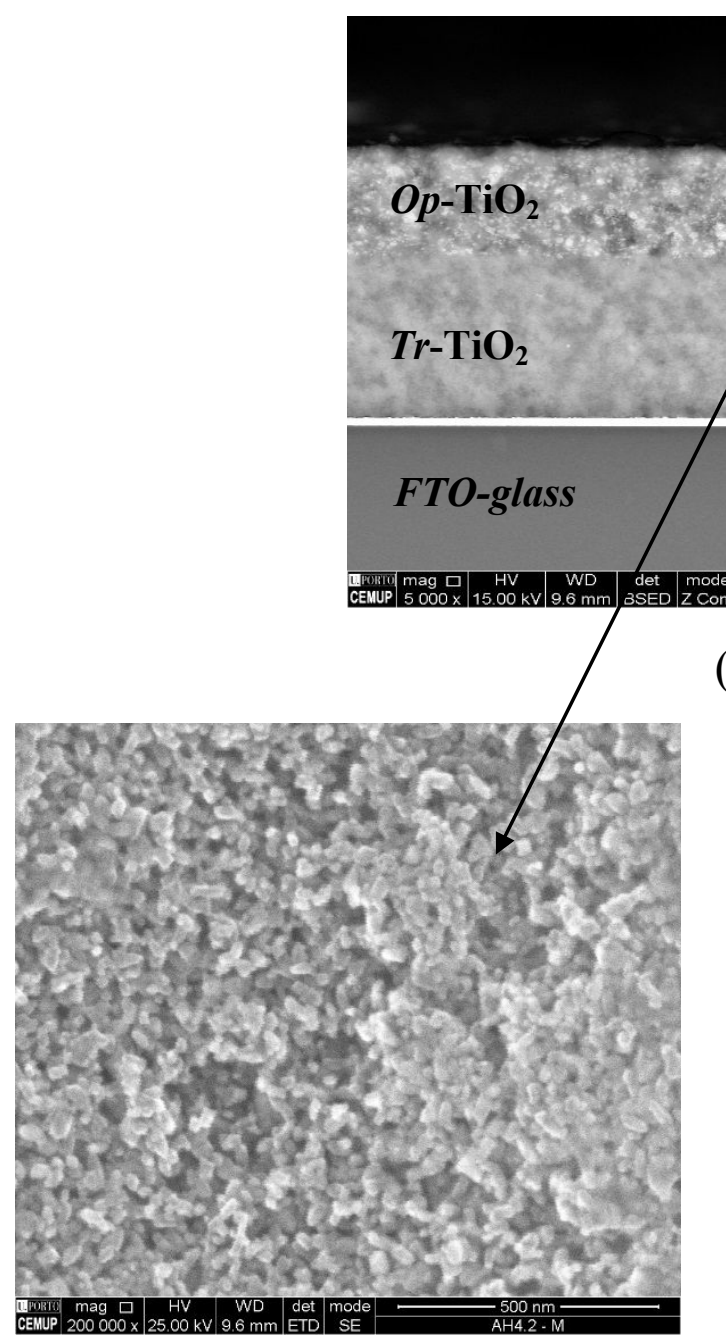

(b) (a)

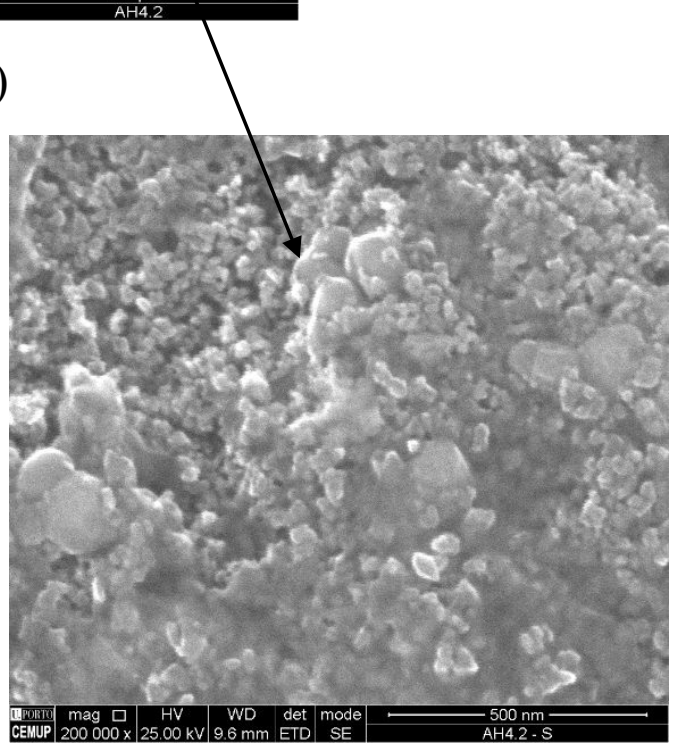

(c)

Figure S2. Typical cross-sectional SEM image of $\mathrm{TiO}_{2}$ film deposited on FTO-glass (a). The film comprises $T r-T i \mathrm{O}_{2}$ layer and $O p-\mathrm{TiO}_{2}$ atop layer; magnified areas from image (a) for $T r-\mathrm{TiO}{ }_{2}$ (b) 
and $\mathrm{Op}-\mathrm{TiO}_{2}(\mathrm{c})$.
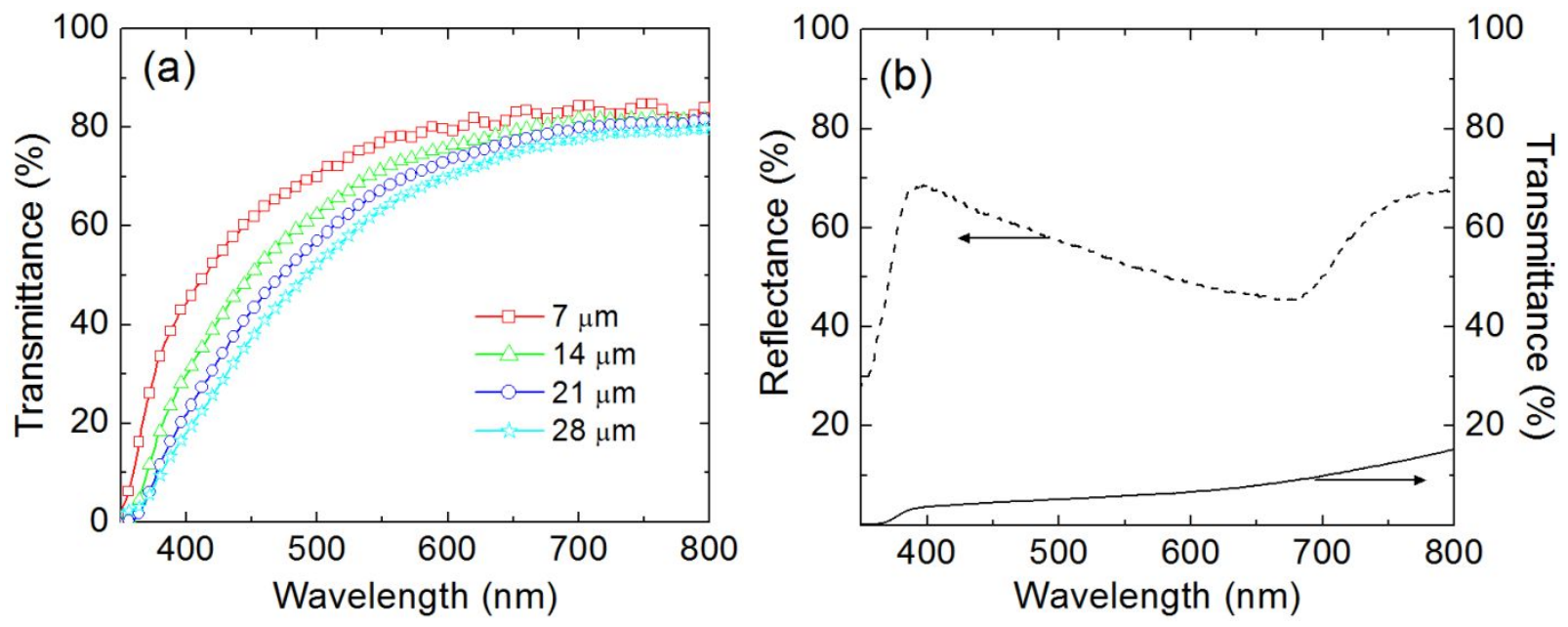

Figure S3. (a) Transmittance of $T r-\mathrm{TiO}_{2}$ layers of different thickness; (b) reflectance and transmittance of $7 \mu \mathrm{m} \mathrm{Op}-\mathrm{TiO}_{2}$ layer.*

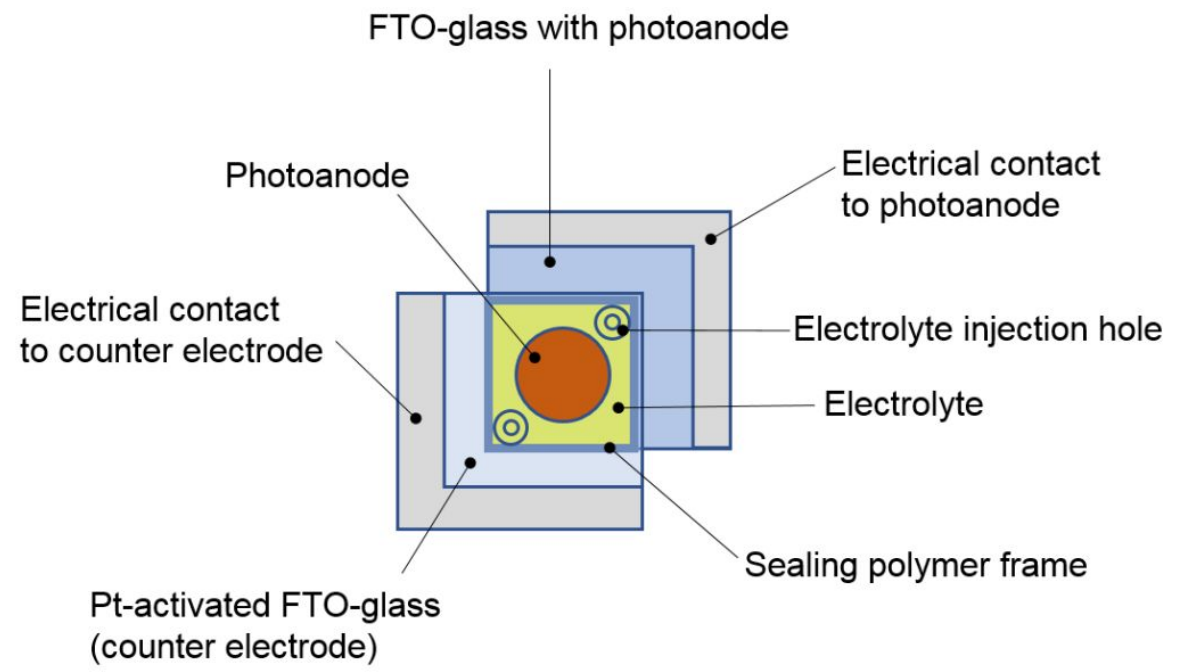

Figure S4. Schematic representation of an assembled DSSC.

${ }^{*}$ The spectra were collected using Shimadzu UV-3600 spectrophotometer equipped with LISR-3100 integrating sphere. Reflectance from $\mathrm{BaSO}_{4}$ powder compact was used as baseline in reflectance spectrum. 


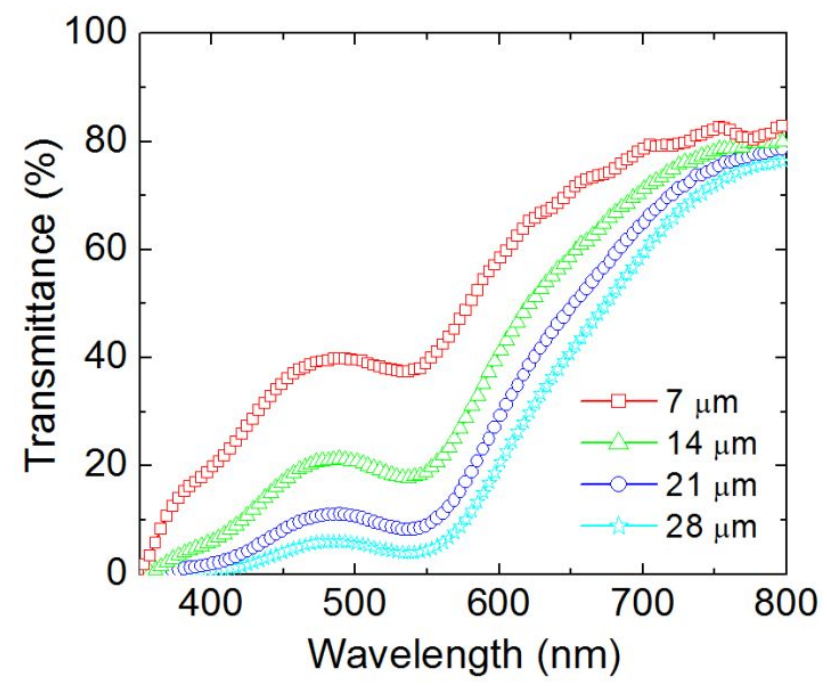

Figure S5. Transmittance spectra of $T r-\mathrm{TiO}_{2}$ layers of different thickness after N719 dye absorption.

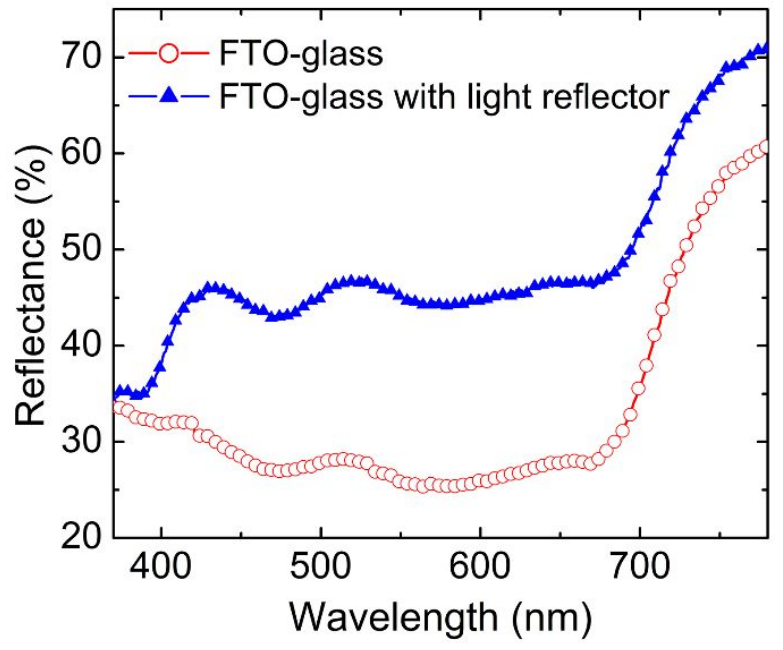

Figure S6. Reflectance spectra of FTO-glass (circles) and FTO glass with PVC light reflecting film on the back site of the glass (triangles). 


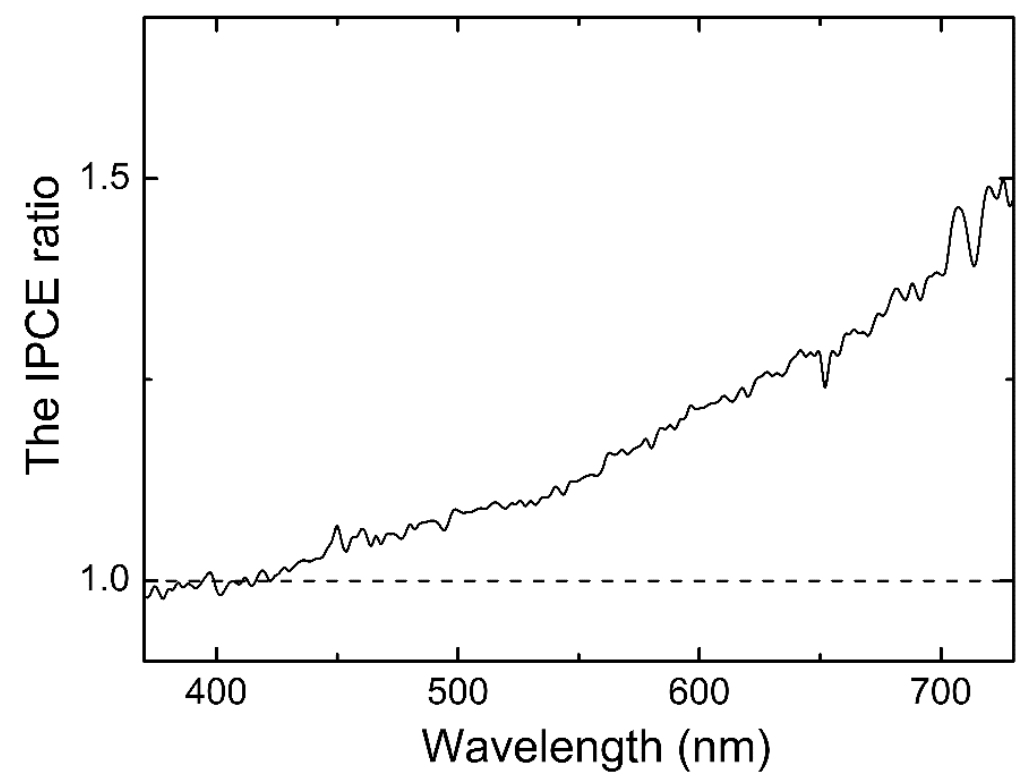

Figure S7. The ratio of IPCE from the cell with light reflector in the back site to the IPCE from the cell without light reflector. 